\title{
McArdle Disease: A Clinical Review
}

Corresponding Author :

R Quinlivan

The Wolfson Centre for Inherited Neuromuscular Disease, RJAH Orthopaedic

NHS Trust, Oswestry, UK, Fax 01691404629

\section{Ros.Quinlivan@rjah.nhs.uk}

Additional authors:

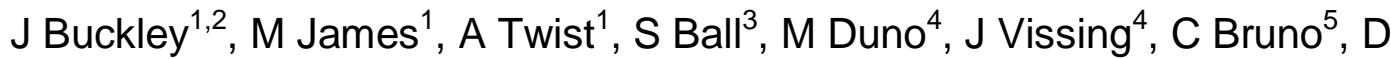

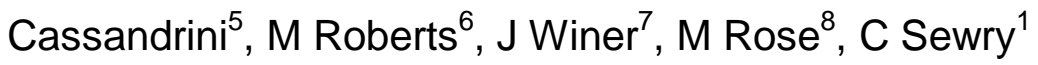

${ }^{1}$ The Wolfson Centre for Inherited Neuromuscular Disease, RJAH Orthopaedic NHS Trust,

Oswestry, UK

${ }^{2}$ The Centre for Exercise and Nutrition Science, University of Chester, UK

${ }^{3}$ The Princess Diana Children's Hospital, Birmingham, UK

${ }^{4}$ The Neuromuscular Research Unit, Departments of Neurology and Clinical Genetics, National University Hospital, Rigshospitalet, Copenhagen, Denmark

${ }^{5}$ The Neuromuscular Diseases Unit, Istituto Giannina Gaslini, Genova, Italy

${ }^{6}$ Greater Manchester Neurosciences Centre, Hope Hospital, Salford, Manchester, UK

${ }^{7}$ Department of Neurology, Queen Elizabeth Hospital, Edgbaston, Birmingham, UK

${ }^{8}$ Department of Neurology, Kings College Hospital \& Kings College School of Medicine, London, UK

Key words: McArdle disease, Glycogen Storage Disorder Type V, Glycogen

Phosphorylase deficiency, PYGM

Word count 4756 


\section{Abstract}

The clinical phenotype of 45 genetically confirmed McArdle patients is described. In the majority of patients (84\%), the onset of symptoms was from early childhood, but diagnosis was frequently delayed until after 30 years of age. Not all patients could recognise a second wind, although it was always seen with exercise assessment. A history of myoglobinuria was not universal, and episodes of acute renal failure had occurred in a minority $(11 \%)$. The condition does not appear to adversely affect pregnancy and childbirth. Clinical examination was normal in most patients, muscle hypertrophy was present in $24 \%$ and mild muscle wasting and weakness was seen only in patients over 40 years of age and was limited to shoulder girdle and axial muscles. The serum creatine kinase (CK) was elevated in all but one pregnant patient. Screening for the mutations p.Arg50X (R50X) and p.Gly205Ser (G205S) showed at least one mutated allele in $96 \%$ of Caucasian British patients, with an allele frequency of $77 \%$ for p.Arg50X in this population. A 12-minute walking test to evaluate patients is described, results demonstrated a wide spectrum of severity with the range of distance walked being 195-1980 metres, the mean distance walked was 512m suggesting significant functional impairment in most patients. 


\section{Background}

McArdle disease (Glycogen storage disease type V, GSDV) is an inherited disorder of glycogen metabolism affecting only skeletal muscle. Brian McArdle described a patient with exercise intolerance who failed to produce lactate during ischaemic exercise [1]. Subsequently, a deficiency of muscle glycogen phosphorylase was identified in patients with McArdle disease [2, 3]. In most affected people, there is no detectable glycogen phosphorylase activity. Traditionally, diagnosis is based upon the inability of the patient to produce lactate during a forearm exercise test, lack of muscle glycogen phosphorylase activity on muscle biopsy and more recently DNA studies to look for variants in PYGM. The ischaemic lactate test has been replaced by a non-ischaemic forearm test, which avoids the potential risk of compartment syndrome, although there may be a greater risk of false positive results $[4,5]$. A functional cycle test has also been shown to be a useful screening assessment, although the equipment required may not be widely available in an outpatient setting [6].

There are three isoenzymes of glycogen phosphorylase, all of which have a high degree of amino acid sequence homology. The muscle isoenzyme is encoded at $11 \mathrm{q} 13$ and is a prerequisite for generation of ATP to fuel contracting muscle [7]. It is found in skeletal muscle and as a hybrid form in the brain and cardiac muscle [8]. There are also liver and brain isoenzymes encoded by separate genes on chromosomes 14 and 20, respectively [9]. The brain isoenzyme is present in foetal muscle and is expressed in regenerating muscle 
fibres $[10,11]$, and is also expressed in smooth muscle. The histochemical method used to localise phosphorylase reveals the activity of all three isoforms.

McArdle disease results from homozygous or compound heterozygous mutations in the myophosphorylase gene (PYGM), on chromosome 11q13. By December 2008, more than 100 mutations had been identified in the gene, which spans 20 exons and many of the mutations are population specific $[12,13,14]$. The most common mutation in Northern Europe and North American patients is a nonsense mutation at p.Arg50X (R50X) in exon 1 (previously referred to as R49X) [15]. A second frequent mutation in the Northern American and Spanish populations is p.Gly205Ser (G205S) [7, 15, 16]. The diagnosis of McArdle disease is confirmed by DNA analysis and/or muscle biopsy with histochemistry and biochemical analysis.

In the resting state and during low-intensity aerobic activity, skeletal muscle primarily utilizes free fatty acids, via oxidative phosphorylation, for energy. Absence of muscle glycogen phosphorylase results in the inability to mobilise muscle glycogen stores. This poses a problem to patients with McArdle disease when there is a need for anaerobic metabolism and a high glycolytic flux for oxidative combustion. Typical symptoms include fatigue, muscle stiffness, myalgia and weakness which is induced by exercise and relieved by rest [17]. If exercise is continued despite these symptoms painful muscle cramping and contracture followed by myoglobinuria occurs. In severe cases, rhabdomyolysis will lead to collapse and reversible acute renal failure requiring dialysis. The 
second wind phenomenon is universal in McArdle disease and occurs due to a switch to alternative fuel substrates required for aerobic metabolism [6].

In McArdle disease, a secondary impairment of oxidative phosphorylation occurs due to a virtual absence of pyruvate, which is normally generated from glycolysis [18]. Without physical training, most individuals will have limited capacity for fatty acid oxidation during exercise $[19,20]$. The effect of this diminished oxidative phosphorylation is a reduction in oxygen consumption to approximately $35 \%$ of normal and a disproportionate exercise-induced increase in heart rate when compared with normal controls [21].

There have been no detailed epidemiological studies of McArdle disease and there is little published data on the natural history of the condition, although several authors have published data on the relative frequency of specific mutations occurring in patients in different European countries [22, 12]. An incidence of 1:100,000 has been reported for the Dallas population (USA) [23], and a small study of neonatal screening for the p.Arg50X mutation in the Netherlands suggested a frequency of 1:350,000 [24]. Amongst affected individuals there is marked heterogeneity in terms of severity, which does not correlate with specific mutations $[26,12,13]$. One possible explanation for variability in expression of the condition could be the co-existence of disease modifying genes such as the Angiotensin converting enzyme gene phenotype (ACE), alpha actinin 3 (ACTN3) and C34T muscle adenosine monophosphate deaminase (AMPD1) [25, 26, 27, 28]. Differences in life-style including dietary habit and fitness are also likely to play a role in variability [29]. 
A specialist clinic for people with McArdle disease was established at The Robert Jones and Agnes Hunt NHS Hospital Trust in 1998. In this study, we present the results of a prospective clinical evaluation of 45 genetically confirmed British patients referred between July 1998 and December 2007.

A total of 55 patients previously diagnosed with McArdle disease were referred to the service, however, in ten of these patients the clinical history was not considered typical for the condition and further investigation including: forearm exercise test, muscle biopsy and PYGM studies failed to confirm the diagnosis. In nine of these patients, there had been a previously reported absence of muscle glycogen phosphorylase on muscle histochemistry and in one patient, the diagnosis of McArdle disease had been made on the basis of an ischaemic forearm exercise test which gave a false positive result. Two of these patients were subsequently diagnosed with Becker muscular dystrophy and one child was diagnosed with mini-core myopathy. In the remaining seven patients the diagnosis was revised to chronic fatigue syndrome (CFS/ME) in accordance with the NICE diagnostic criteria for CFS/ME [30].

\section{Methods}

The forty-five remaining patients were included in a prospective clinical evaluation which included a detailed structured and standardised history, clinical examination, functional assessment and investigations including: serum CK, plasma urate and molecular genetic studies for mutations in PYGM, including cDNA studies from whole muscle where appropriate. Structured clinical history 
included: the patient's first memory of exercise intolerance (where possible this was corroborated by a first degree relative), the age at which diagnosis was confirmed and the predominant symptom which caused the person to first seek medical attention (although this might not necessarily have related to the consultation that led to diagnosis), specific questions relating to the second wind phenomenon, myoglobinuria and acute renal failure were also asked.

Results from previous investigations including forearm exercise tests and muscle pathology were not included in the data collection, these investigations had been performed at many different centres across the UK and at different times (sometimes many years earlier) as a consequence reliable and accurate data might have been difficult to obtain, however, in all subjects the diagnosis was confirmed by molecular analysis.

The resulting information was anonymised and recorded onto a secure database and analysed separately by two individuals. Approval for the clinical evaluation study was given by the Hospital's audit committee. Approval was obtained from the Shropshire ethics committee to develop a novel functional 12minute exercise assessment, and informed written consent was obtained from the participants.

We recognised that a pragmatic functional assessment would be necessary to monitor individual patient progress on a routine outpatient basis, hence the devising of a modified 12-minute walking test to assess functional ability. This was combined with using a Borg's CR-10 scale [34] to rate muscle sensations (fatigue, tightness, discomfort, cramping and pain) on a 10 point 
scale. We chose the CR-10 scale for rating perceived muscle pain (RPP), rather than Borg's traditional scale of perceived exertion (RPE) for two reasons, firstly it has previously been used in conjunction with a timed walking assessment as a primary outcome measure for a clinical trial in McArdle disease [35] and secondly because the RPE scale was designed to measure the linear responses of overall aerobic body work, whereas the CR-10 scale was designed to have congruence with the psychophysical phenomenon of the curvilinear responses in perceptions to specific muscle sensations during exertion $[34,35]$. Twenty of the 45 patients were recruited for this study following informed written consent.

Each subject was asked to walk as far as they could in 12 minutes and to rate muscle sensation using the RPP scale, subjects were instructed not to allow their RPP score to exceed level 4 (moderate sensation). Heart rate was recorded via a wireless chest strap transmitter (Polar, Kempele Finland) and the total distance walked was recorded. Walking distance and thus speed, heart rate and RPP were recorded each minute. Differences in minimum and peak values of walking speed, heart rate and muscle pain over the 12 minutes of the test were assessed using a one-way analysis of variance.

\section{Results}

Age of onset and age at diagnosis

There was an equal preponderance of males $(n=22)$ and females $(n=23)$. 
Their ages ranged from 9-64 years. Table 1 summarises the age of onset of symptoms and the age at which diagnosis was made. The majority $(n=38,84 \%)$ of patients recalled symptoms before 10 years of age, whereas only seven (15\%) patients were diagnosed by 10 years of age. By contrast, 22 patients $(49 \%)$ were diagnosed after 30 years of age. Most individuals reported that their symptoms in childhood had been attributed to "growing pains" and not investigated further. Symptoms

The features identified from patient histories are summarised in table 2 . The most frequent symptom that precipitated $44 / 45$ of the subjects to seek medical attention was painful muscle cramping, with or without contracture, which involved any skeletal muscle including: proximal and distal limb, paraspinal, chest and jaw muscles. One subject presented with recurrent hospital admission due to episodes of unexplained collapse associated with myoglobinuria. He was subsequently diagnosed with juvenile myoclonic epilepsy, his myoglobinuria having been triggered by seizures. He denied any significant muscle symptoms other than a mild discomfort occurring with exercise. A faint trace of glycogen phosphorylase (rather than complete absence) was seen in his muscle histochemistry, there were no regenerating muscle fibres. Genomic DNA studies showed him to have two PYGM sequence variants: p.Arg50X mutation and a previously undescribed variation, c. $2430 \mathrm{C}>\mathrm{T}$. cDNA studies from whole muscle suggested this novel variant was likely to create an aberrant splice site [31]. Cryptic splice mutations can occasionally be leaky allowing a minor fraction of correct splicing, which explains the milder phenotype [32]. 
Recognition by patients of a second wind, although typical for the condition, was absent in $22 \%(n=10)$, and severe contracture with myoglobinuria had never occurred in $38 \%(n=17)$. At least one episode of acute renal failure necessitating haemodialysis had occurred in five patients (11\%). Disabling central and peripheral fatigue with poor memory and concentration, resembling chronic fatigue syndrome, was relatively common, affecting $40 \%$ of all patients. A significant proportion of patients (31\%) had been treated at some time for anxiety and/or depression.

Two patients had come to medical attention following a reported increase in frequency of muscle cramps after statin treatment had been prescribed for hypercholesterolaemia. It remains unclear whether statin therapy actually worsened symptoms or merely led to the diagnosis being unmasked because of routine CK monitoring. Five patients (11\%) had raised plasma urate levels and had been prescribed allopurinol for symptomatic gout; one patient had a single episode of renal calculi secondary to urate nephropathy. Affected adults (both male and female) reported muscle cramps +/-myoglobinuria, as a consequence of sexual intercourse. Most patients reported a sedentary lifestyle due to fear of developing rhabdomyolysis and acute renal failure.

Three patients had been treated with opiates prior to referral; two had become dependent on oral morphine and were unable to withdraw treatment. All three reported severe exercise intolerance due to incapacitating chronic pain, muscle cramps and myoglobinuria despite continuous opioid treatment. Physical Examination 
The majority $(71 \%, \mathrm{n}=32)$ of patients were overweight with a BMl greater than 26. Facial weakness was not seen in any patient. Muscle hypertrophy was seen in eleven patients (24\%) affecting all or some of the following muscle groups: deltoid, biceps, calves and thighs. Mild muscle wasting predominantly involving the paraspinal, peri-scapular and proximal upper limb muscles was present in fifteen patients (33\%). Mild weakness (grade 4/5 MRC scale) involving axial and shoulder girdle muscles was seen in seven patients (16\%), all of whom were over 40 years of age. Lower limb wasting and weakness was not a feature in the McArdle patients, by contrast quadriceps wasting and weakness was seen in the two wrongly diagnosed patients who had Becker muscular dystrophy.

Progressive muscle wasting and limb girdle weakness was seen in one patient with homozygous p.Arg50X mutations. The patient had marked calf hypertrophy and pes cavus, which was not seen in any other McArdle patient. In addition to complete absence of muscle glycogen phosphorylase in most fibres, his muscle biopsy showed atypical pathological features with several regenerating fibres with activity of the brain isoform of phosphorylase and several rimmed vacuoles. Immunolabelling of sarcolemmal proteins affected in various forms of muscular dystrophy was normal (figure 1a, figure 1b shows typical histology for comparison). The patient is thought to also be affected with a limb girdle muscular dystrophy. DNA studies for Xp21, FKRP, SMN, GNE and caveolin 3 showed no abnormality. Further genetic studies are pending.

Investigations 
The serum CK was raised in all but one female patient who was 32 weeks pregnant at the time of testing, mean serum CK was 2,471 iu/l (ranging from 85$12,405 \mathrm{iu} / \mathrm{l}$, normal values $<190 \mathrm{iu} / \mathrm{l})$. Repeat testing of serum CK in the pregnant patient, two years after the birth of her child was raised at 1384iu/l.

Molecular genetic studies undertaken to look only for the mutations p.Arg50X and p.Gly205Ser as an initial screen were undertaken at The Princess Diana Children's Hospital, Birmingham. Forty three patients, all of whom were of British Caucasian origin, carried at least one allele mutated for p.Arg50X (95\%) (table 2). This initial screen confirmed the diagnosis in $66 \%$ of patients, who were shown to be either homozygous or compound heterozygotes. One father and daughter demonstrated pseudo-dominance; both were homozygous for p.Arg50X mutations. There was no consanguinity, the wife/mother was heterozygous for p.Arg50X.

Full gene sequencing was undertaken on DNA from patients shown to be either heterozygotes or who had neither allele mutated. Two patients did not carry either of the p.Arg50X or p.G205Ser mutations. One was of Pakistani origin and was found to carry a novel homozygous mutation in exon 1 (c.14delT), the other was a caucasian female patient and was found to be a compound heterozygote for p.Leu36Pro and c. $1239+1 \mathrm{G}>\mathrm{A}$. In the remaining 13 patients the second mutation was scattered across the PYGM gene, nine of which were novel mutations [31] and are shown in table 3. A detailed account of the methods used for molecular genetic analysis is reported by Duno et al 2009 [31]. 


\section{Functional testing}

Patients own perceptions of exercise tolerance were variable ranging from ability to walk only 10 yards on the flat to normal levels of activity (including regular sporting activities and hill walking). Each subject was asked to walk as far as they could in 12 minutes and to rate muscle sensation using the RPP scale, subjects were instructed not to allow their RPP score to exceed level 4 (moderate sensation). Heart rate was recorded via a wireless chest strap transmitter (Polar, Kempele Finland) and the total distance walked was recorded. Walking speed, heart rate and RPP were recorded each minute. Differences in minimum and peak values of walking speed, heart rate and muscle pain over the 12 minutes of the test were assessed using a one-way analysis of variance.

All 20 patients completed the assessment with no episodes of severe cramp or rhabdomyolysis (assessed by laboratory biochemical analysis for CK and urine myoglobin concentrations). In the first six minutes of assessment, RPP ratings and heart rate $(\mathrm{HR})$ increased significantly from the resting value $(p<0.0005)$, whilst walking speed decreased significantly $(p=0.013)$ (figure $2 \mathrm{a}$,

2b, 2c). Significantly correlated perturbations in RPP and HR occurred between 1 and $6 \min (r=0.521 ; p=0.019)$ and 6 and $11 \min (r=0.678 ; p=0.001)$.

Between 6 and 11 min, RPP decreased $(p=0.011$ ), walking speed increased ( $p$ $=0.017)$, thus demonstrating a second wind phenomenon.

The total distance walked in 12 minutes was highly variable ranging from 195-1980 metres, the mean distance walked was $512 \mathrm{~m}$, indicating a wide range of functional variability. There was no significant difference between the median 
distances walked by p.Arg50x heterozygotes (850m) compared with p.Arg50x homozygous participants $(920 \mathrm{~m})$ (Wilcoxon rank sum, $\mathrm{W}=75.5, \mathrm{n} 1=9$, n2 $=11$; $\mathrm{p}=.149$ ), thus suggesting no genotype/phenotype correlation. Figure $2 \mathrm{c}$ illustrates that the test was of a suitable intensity in keeping with recommended intensities of aerobic exercise [40], where throughout the test, with exception to the first minute, heart rates were above $65 \%$ of maximum. Figure 3 , additional data from a further 34 patients assessed as part of routine clinic follow up, shows a relatively even distribution of walking distance across a wide range of functional abilities, with a small peak of participants in the $800 \mathrm{~m}$ category. In this group the range was $159 \mathrm{~m}-1180 \mathrm{~m}$, with a mean distance of $669.3 \mathrm{~m}$, demonstrating similar results to the original research study.

\section{Pregnancy}

Data were based upon reported experience from affected females, and suggests that McArdle disease does not significantly increase the risk of complications for pregnancy and delivery. Fourteen women had given birth to 21 children. Two women reported worsening muscle cramps during pregnancy but the rest reported feeling better than usual. There was one twin delivery, normal birth was achieved in 17/21; two babies were born by forceps delivery and two by emergency caesarean section, one child was born following elective caesarean section because of a previous section. The rate of interventional delivery was $15 \%$, which does not differ significantly from healthy women [36]. 
One woman recalled 'mild' myoglobinuria following the delivery of her third child, but no specific intervention was required.

\section{Discussion}

Following the development of a specialist clinic for McArdle disease an unexpected finding was the relatively high frequency of false diagnosis where 10 out of 55 patients previously diagnosed did not have the condition. Two of the patients wrongly diagnosed with McArdle disease had Becker muscular dystrophy, both had presented with exertional cramps and myoglobinuria. Unlike the McArdle patients they both had quadriceps wasting and significant pelvic girdle weakness. Seven patients had chronic fatigue syndrome, unlike the McArdle patients, none of these individuals complained of exercise related muscle cramps and all had a normal serum CK and ischaemic lactate test. In these patients the diagnosis of McArdle disease had been previously made on the basis of muscle biopsy demonstrating absent phosphorylase activity. A repeat muscle biopsy, however, did not confirm this finding.

The commonly used histochemical method is specific for muscle glycogen phosphorylase and in McArdle disease it fails to stain in mature skeletal muscle fibres. In regenerating muscle fibres and smooth muscle fibres present in blood vessels it will stain positive because of presence of the brain isoenzyme, this is a useful indicator that the method has worked. The method relies on activation and utilisation of the enzyme in reverse direction (glycogen synthesis) leading to the accumulation of glycogen, which is visible as iodine-reactive material. The colour 
development is not stable in aqueous mountants and it is essential to include a control sample in parallel with the test sample and to observe the outcome as soon as possible to prevent a false diagnosis [7]. If, however, the sections are dehydrated and mounted in resins the colour survives well and this can avoid a false negative result. [7]. A false diagnosis of McArdle disease can also potentially occur in critically ill patients who have secondary glycogen depletion, muscle histochemistry will appear negative for glycogen phosphorylase [37]. On the other hand, diagnosis can be missed if muscle biopsy is undertaken too soon after massive rhabdomyolysis. Although the regenerating fibres express the brain glycogen phosphorylase isoenzyme staining may appear weak. Under such circumstances it is wise to delay muscle biopsy until at least one month after the patient has fully recovered muscle function.

The diagnosis of McArdle disease can be confirmed by sequencing PYGM for mutations, although this is time consuming and costly. Screening for the mutations p.Arg50X and p.Gly205Ser before undertaking full gene sequencing is especially useful for British Caucasian patients; it is cost effective and may prevent the need for a muscle biopsy and full gene sequencing. In this cohort, the allele frequency of the p.Arg50X mutation was $77 \%$. This confirms previous studies, which have shown the p.Arg50X mutation to be most prevalent in British and North American patients [14,15].

In this series, there was an equal preponderance of male and female patients as would be expected for an autosomal recessive condition. However, a previous retrospective review reported a predominance of affected males and 
concluded that this probably reflected a gender related reporting bias, since males are more likely to undertake strenuous recreational and occupational activities [17]. The onset of symptoms most commonly occurred in early childhood, but diagnosis was delayed until after 30 years of age in more than half of the patients. The long delay from onset of symptoms to diagnosis despite patients seeking medical help suggests that primary care physicians are not familiar with the condition.

For many of the patients, memories of school years were unfavourable, their symptoms having been attributed to 'growing pains' and not investigated further. In children, idiopathic leg pains are common, they usually occur at night, are not exercise-related and the serum CK is normal. By contrast, the serum CK is almost always raised in McArdle disease. Any patient presenting with a history of muscle cramps affecting any skeletal muscle during exercise should lead the doctor to suspect a metabolic myopathy. A history of painful cramps that occur within a few minutes of initiating exercise and which subside rapidly with rest in conjunction with a raised serum creatine kinase is highly suggestive of McArdle disease. If the individual continues to exercise to the point of contracture, there will be a history of painful rigidity, which may persist for several hours, this will almost always be accompanied by muscle swelling and myoglobinuria. The history of a second wind should be specifically looked for, but may not always be recognised by patients, however, during exercise assessment the second wind is a universal finding [6]. A history of myoglobinuria is not universal, and its absence does not exclude the condition. 
In this series, significant muscle hypertrophy was present in $24 \%$ of patients; a more frequent finding than previously reported which was $10 \%$ in a cohort of 34 patients [13]. The aetiology of this finding is unclear. Mild muscle wasting affecting the paraspinal muscles and shoulder girdle was relatively common. Muscle weakness, when present, was mild (not worse than MRC grade 4) and predominantly involved the shoulder girdle and axial muscles. Muscle weakness was not seen in any patient who was under 40 years of age. Significant shoulder girdle muscle atrophy and weakness has been previously described in an elderly McArdle patient [32]. The presence of significant lower limb wasting and weakness was not a typical feature and alerted us to look carefully for an alternative diagnosis, which in this case, was Becker muscular dystrophy.

As with previous studies we found no genotype-phenotype correlation [12, 13]. In our research cohort, the 12-minute walk test demonstrated a wide range of variability (195-1980m), however, the mean distance walked in 12 minutes was $512 \mathrm{~m}$. Healthy sedentary individuals would be expected to walk more than $1200 \mathrm{~m}$ in 12 minutes. McArdle patients who walk less than $500 \mathrm{~m}$ in 12 minutes are most likely to have significant impairment in performing activities of daily living (ADL). The average walking speed of $41.7 \mathrm{~m} / \mathrm{min}(2.5 \mathrm{~km} / \mathrm{hr})$ in the research cohort, represents an oxygen uptake of $8-10 \mathrm{ml} / \mathrm{kg} / \mathrm{min}$ (2-3 metabolic equivalents) [39], most ADLs range between 2-4 metabolic equivalents [40], which means that the McArdle patients achieving less than 500m were struggling 
to sustain what healthy individuals would consider to be a very low exercise intensity.

People with McArdle disease are afraid to exercise for fear of developing rhabdomyolysis and acute renal failure, however, acute renal failure was a relatively infrequent event and can be avoided by pacing exercise intensity for the first few minutes of activity. Most patients adapt to their condition by adopting a sedentary life-style, the consequence of which will be de-conditioning and may lead to exacerbation of fatigue and exercise intolerance. Weight gain is common, with the majority of patients being overweight or obese, this is likely to worsen symptoms by increasing dependence on glycolytic metabolism during exercise. Psychological disturbance was a relatively common finding, and may reflect a combination of living with limited exercise intolerance and possibly diagnostic delay. Earlier diagnosis, improved management and a greater awareness of the condition could potentially improve quality of life.

In an open study of aerobic exercise training in people with McArdle disease, Haller et al demonstrated a significant improvement in work capacity and no serious untoward effects [20]. The authors concluded that the mechanism of action was likely to be an improvement in mitochondrial metabolism secondary to an increased delivery of blood borne mitochondrial fuels through improved blood flow [20]. Thus, aerobic exercise is safe for people with McArdle disease and should be recommended for all patients, regardless of age or severity [20, $41,42]$. Advice should be given on how to avoid isometric muscle contraction 
and anaerobic exercise which are dangerous for patients with McArdle disease and are likely to lead to contracture and rhabdomyolysis.

In this series, three patients taking regular opiate medication were the most disabled and complained of incapacitating chronic pain with frequent episodes of contracture and myoglobinuria. There was no evidence to suggest symptomatic benefit from opiates, in fact it is possible that this strong form of analgesia masks mild to moderate discomfort, the normal warning sign to stop exercising before a cramp occurs, and thus could potentially increase the frequency of episodes of contracture and rhabdomyolysis.

The potential risk for adverse reaction to statins remains uncertain. There are two published case reports of worsening cramps following statin treatment and one case report of acute rhabdomyolysis with Ezetimibe, although the patient had also exercised prior to the episode $[42,43,44]$. In this series, two patients were diagnosed after a statin was prescribed. It remains unclear as to whether or not the condition was made worse by statins or merely unmasked in these patients. When considering statin treatment in people with McArdle disease, cautious introduction of treatment with close monitoring is advisable until further evidence becomes available on the effect of statins in the condition.

In this series there was an increased risk of symptomatic gout. One patient also had a renal calculus, probably secondary to a urate nephropathy. This is probably due to a secondary increase in purine metabolism [45]. The findings suggest that plasma urate levels should be monitored in patients with McArdle disease. Women can be reassured that a normal pregnancy and 
delivery is possible.

There is no cure for McArdle disease, and as yet no specific treatment can be recommended [29]. Low dose creatine was shown to confer a small benefit in a small number of patients [46]. Ingestion of sucrose prior to planned exercise improves performance [47], but is not a useful treatment for routine daily living. In one series, a diet rich in carbohydrate resulted in better exercise performance than a protein rich diet [48]. Teaching patients a strategy to achieve a second wind enables them to exercise safely.

A functional exercise assessment is necessary to monitor the effectiveness of any future clinical intervention. Traditionally exercise capacity in McArdle disease is assessed using cycle ergometry which has been demonstrated to be reliable for physiological and treatment studies [42]. However, the lack of availability of sport's equipment in many clinical settings makes this test impractical for widespread routine outpatient use. Furthermore, walking is the most common and functional form of physical activity and it seems sensible to have a simple test that represents this activity. The assessment clearly demonstrated the presence of a second wind between 6 and 10 minutes, in keeping with previous studies [24], where walking performance clearly improved either by a reduction in discomfort for a given walking speed or that for any given heart rate, walking speed was increased. This assessment additionally acts as an education tool because it enables patients to experience and clearly see and understand the concept of a second wind. Finally, a record of the total distance walked in 12 minutes can be utilised as both a functional status 
reference point and as a clinical outcome measure as an estimate of functional aerobic fitness.

Acknowledgements: The authors would like to thank Mr Andrew Wakelin of the Association for Glycogen Storage Disorders UK (AGSDUK) who gave feedback on the manuscript and Professors Roger Eston (University of Exeter) and Julius Sim (Keele University) who gave analytical advice. Financial support was given by the AGSD (UK) and The Muscular Dystrophy Campaign

Competing Interests: None declared

\section{Licence for publication:}

The Corresponding author has the right to grant on behalf of all authors and does grant on behalf of all authors, an exclusive licence (or non exclusive for Government employees) on a worldwide basis to the BMJ Publishing Group Ltd to permit this article (if accepted) to be published in JNNP and any other BMJPGL products and sublicences such use and exploit all subsidiary rights as set out in our licence. (http://group.bmj.com/products/journals/instructions-forauthors/licence-forms) 


\section{References:}

1. McArdle B. Myopathy due to a defect in muscle glycogen breakdown. Clin Sci. $1951 ; 10: 13-33$

2. Mommaerts WFH, Illingworth B, Pearson CM, Guilorg RJ, Seraydarian K. A functional disorder of muscle associated with the absence of phosphorylase. Proceedings of the National academy of Science USA 1959; 3: 18-22

3. Schmidt R, Mahler R. Chronic progressive myopathy with myoglobinuria: demonstration of a glycogenolytic defect in muscle. J Clin. Investigation 1959; 108: 2044-58

4. Hogrel JY. Laforet P. Ben Yaou R. Chevrot M. Eymard B. Lombes A. A non-ischemic forearm exercise test for the screening of patients with exercise intolerance. Neurology; 56(12):1733-85.

5. Kazemi-Esfarjani P. et al A non-ischaemic forearm exercise test for McArdle disease. Ann Neurol 2002: 56; 1779-1780

6. Vissing J. Haller RG. A diagnostic cycle test for McArdle's disease. Ann Neurol. 54(4):539-42, 2003

7. Beynon R J, Quinlivan RCM, Sewry C A. Selected disorders of carbohydrate metabolism. In: Structural and molecular basis of skeletal muscle disease p.182-188. Editor George Karpati. ISN Neuropath Press 2002

8. Crerar M, Karlsson O, Fletterick R, Hwang P. Chimeric muscle and brain glycogen phosphoryalses define protein domains governing isozyme- 
specific responses to allostearic activation. J Bio. Chem. 1995; 270;

$13748-13756$

9. Newgard C, Hwang PK, Fletterick RJ. The family of glycogen

phosphorylases: Structure and function. J Critical Reviews in Biochemistry and Molecular Biology 1989; 24: 69-995.

10. DiMauro S, Arnold S, Miranda A, Rowland LP. McArdle disease: The mysterious appearance of phosphorylase activity in cells that ought to lack the genetic program. A foetal isoenzyme? Trans Am Neurol Assoc 1977; 102: $112-5$

11. Martinuzzi A, Schievano G, Nascimbeni M, Fanin M. McArdle's disease. The unsolved mystery of the reappearing enzyme. Am. J Pathology 1999; $154: 1893-1897$

12. Deschauer M, Morgenroth A, Joshi PR, Glaser D, Chinnery PF, Aasley J et al. Analysis of spectrum and frequencies of mutations in McArdle disease: Identification of 13 novel mutations. J Neurol 2007; 254; 797-802 13. Aquaron R, Berge-Lefranc JI, Pellissier JF, Montford MF, Mayan M, Figarella-Branger D. Molecular characterisation of myophosphorylase deficiency (McArdle disease) in 34 patients from Southern France: identification of 10 new mutations. Absence of genotype-phenotype correlation. Neuromuscular Disorders 2007: 17; 235-41

14. Bartram C, Edwards RHTE, Clague J, Beynon R. McArdle's disease a nonsense mutation in exon 1 of the muscle glycogen phosphorylase gene explains some but not all cases. Hum. Mol. Genet 1993; 2: 1291-3 
15. Tsujino S, Shankse S, Di Mauro S. Molecular genetic heterogeneity of myophosphorylase deficiency (McArdle's Disease). N Eng J Med 1993;329: $241-5$

16. Andreu A, Nogales-Gadea G, Cassandrini D, Arenas J, Bruno C. McArdle Disease: Molecular genetic update. Acta Myologica 2007; XXV1; 53-57

17. DiMauro S, Hays A, Tsujino S. Metabolic Disorders Affecting muscle; Non Lysosomal Glycogenoses. In Myology Ed A Engel 2004 p1537-1582

18. Haller RG, Lewis SF, Cook JD, Bloomvist CG. Myophosphorylase activity impairs muscle oxidative metabolism. Annals Neurology 1985; 17:196-9

19. De Stefano N, Argov Z, Matthews P.M, Karpati G., Arnold D.L. Impairment of muscle mitochondrial oxidative metabolism in McArdle's Disease. Musc. Nerve 1996; 19: 764-769

20. Haller R G, Wyrick P, Taivassalo P, Vissing J. Aerobic conditioning: an effective therapy in McArdle's disease. Ann. Neurol 2006; 59: 922-8

21. Vissing J, Vissing S.F, Maclean D, Saltin B, Quistorff B, Haller R.J.Sympathetic activation in exercise is not dependent on muscle acidosis direct evidence from studies in metabolic myopathies. J Clin. Invest. 1998; 101 (8): 1654-66

22. Bruno C, Cassandrini D, Martinuzzi A, Toscano A, Moggio M, Morandi L, Servidei S, Mongini T Angelini C, Musemeci O, Comi GP, Lamperti C, Filosto M, Zara F, Minetti C. McArdle disease: the mutation spectrum of PYGM in a large Italian cohort. Hum. Mut. 2006; 27: 718 23. Haller RG. Treatment of McArdle Disease. Arch Neurol 2002; 57: 923-4 
24. van Elfen N, de Bie HJJ, Wevers RA, Arenas J, van Engelen BGM: The prevalence and genetic characteristics of McArdle's disease in The Netherlands. Neuromuscular Disorders 2002:12; 752.

25. Gomez-Gallego F, Santiago C, Moran M, Mate-Munoz JL, Fernandez Del Valle $\mathrm{M}$ et al. The I allele of the ACE gene is associated with improvement in exercise capacity in women with McArdle disease. BJSM 2008

26. Lucia A, Gomez-Gallego F, Santiago C, Perez M, Mate-Munoz JL, Chamorro-Vina C et al The 577X allele of the ACTN3 gene is associated with improved exercise capacity in women with McArdle's disease. Neuromuscular Disorders 2007; 17 (8): 603-10

27. Martinuzzi A, Sartori E, Fanin M, Nascimbeni A, Valente L, Angelini C et al. Phenotype modulators in myophosphorylase deficiency. Annals of Neurology 2003; 53 (4): 497-502

28. Rubio JC, Gomez-Gallego F, Santiago C, Garcia-Consuegra I, Perez M, Barriopedro Ml et al. Genotype modulators of clinical severity in McArdle disease. Neuroscience Letters 2007; 422(3): 217-222

29. Quinlivan R M, Martinuzzi A, Beynon R J: Pharmacological and nutritional treatment of McArdle's disease (Glycogen storage disease V) Cochrane Database Syst. Rev. 2008; 2: CD003458

30. National Institute for Health and Clinical Excellence. Chronic Fatigue Syndrome/myalgic encephalomyelitis (or encephalopathy): diagnosis and management of chronic fatigue syndrome/myalgic encephalitis (or encephalopathy) in adults and children. CG 53 Aug 2007 
31. Duno M. Quinlivan R. Vissing J. Schwartz M High-resolution melting facilitates mutation screening of PYGM in patients with McArdle disease. Annals of Human Genetics 2009;73:292-7

32. Vissing J, Duno M, Schwartz M, Haller RG .Splice mutations preserve myophosphorylase activity that ameliorates the phenotype in McArdle disease. Brain. 2009 p1545-52

33. Borg, G. Borg's Perceived Exertion and Pain Scales, Champaign, IL: Human Kinetics. 1998

34. Lane RJ. Turnbull DM. Welch JL. Walton J. A double-blind, placebocontrolled, crossover study of verapamil in exertional muscle pain. Muscle \& Nerve. 9(7):635-41, 1986 Sep

35. Grant S, McMillan, Newell J, Wood L, Keatley S, Simpson D, Leslie K, Fairlie-Clark S. Reproducibility of the blood lactate threshold, $4 \mathrm{mmol}-\mathrm{I}^{-1}$ marker, heart rate and ratings of perceived exertion during incremental treadmill exercise in humans. Applied Physiology 2002: 87; 159-66

36. Nice $\mathrm{C}$ et al. A prospective audit of wound-infection rates after Caesarean section in 5 West-Yorkshire hospitals. J Hosp Infect 1996;33(1):55-61.

37. Pacheco M.C, Miles L, Bove K. False negative histochemical reaction for myophosphorylase activity in fulminant sepsis due to methicillin resistant staphylococcus aureus. Neuromusc. Dis. 2007;17: 983-985

38. Wolfe GI, Baker NS, Burns RG, Barohn RJ. McArdle's disease presenting with asymmetric, late onset arm weakness. Muscle and Nerve 2000;23: $641-5$ 
39. ACSM's Guidelines for Exercise Testing and Prescription ( $7^{\text {th }}$ Edition). Lippincott Williams and Wilkins, Baltimore ML, 2005Ainsworth BE, Haskell WL, Whitt MC, Irwin ML, Swartz AM, Strath SJ, O'Brien WL, Bassett DR Jr, Schmitz KH, Emplaincourt PO, Jacobs DR Jr, Leon AS. Compendium of physical activities: an update of activity codes and MET intensities. Med Sci Sports Exerc. $2000 ; 32(9$ Suppl): S498-504

40. Martin P, Rubio M, Mate-Munoz J, Gomez-Gallego J, Foster F, Andreu C, Arenas A, Luci J, Fleck A. Exercise capacity in a 78 year old patient with McArdle disease: it is never too late to start exercising. British J Sports Med 2006: 40; 725-6

41. Mate-Munoz J, Moran M, Perez M, Chamorro-Vina C, Gomez-Gallego F, Santiago C, Chicharro L, Foster C, et al. Favourable responses to acute and chronic exercise in McArdle patients. Clinical J Sports Med 2007: $17 ; 297-303$

42. Livingstone C, Riyami A, Wilkins S, Ferns P, Gordon A. McArdle's disease following statin-induced myositis. Ann. Clin. Biochem. 2004; 41: 338-40

43. Barth JH, Brownjohn AM, Jamieson DRS. The case of stainless statins Annals Clin. Biochem. 2003; 40: 576-7

44.Perez-Calvo J, Civeira-Murillo F, Cabello A. Worsening myopathy associate with ezetimibe in a patient with McArdle disease. QJ Med; 2005: $461-464$

45. Mineo I, Kono N, Hara N, Shimizu T, Yamada Y, Kawachi M, Klyokawa H, Wang YL, Tarui S. Myogenic Hyperuricaemia. A common 
pathophysiologic feature of glycogenosis types III, V, VII. New England J. Medicine 1987: 1987; 317: 75-80

46. Vorgerd M, Grehl T, Jager M, Muller K, Freitag G, Patzold T et al.Creatine therapy in myophosphorylase activity (McArdle's disease). A placebo controlled crossover trial. Archives of Neurology 2000; 57: 956-63

47. Vissing J, Haller RG. Effect of oral sucrose on exercise intolerance in patients with McArdle's Disease. New Eng J Med 2003; 349: 2503 48. Andersen ST, Vissing J. Carbohydrate- and protein-rich diets in McArdle disease: Effects upon exercise capacity. J Neurol. Neurosurg Psychiatry 2008; 79: 1359-63 


\section{Appendix}

Table 1

\begin{tabular}{|l|l|l|l|l|l|l|}
\hline Age (years) & $<10$ & $10-19$ & $20-29$ & $30-39$ & $40-49$ & $>50$ \\
\hline $\begin{array}{l}\text { Age of onset } \\
\mathrm{n}=45\end{array}$ & $\begin{array}{l}38 \\
(84 \%)\end{array}$ & $\begin{array}{l}5 \\
(11 \%)\end{array}$ & & $\begin{array}{l}2 \\
(5 \%)\end{array}$ & 0 & \\
\hline $\begin{array}{l}\text { Age at diagnosis } \\
\mathrm{N}=45\end{array}$ & $\begin{array}{l}7 \\
(15 \%)\end{array}$ & $\begin{array}{l}8 \\
(18 \%)\end{array}$ & $\begin{array}{l}8 \\
(18 \%)\end{array}$ & $\begin{array}{l}9 \\
(20 \%)\end{array}$ & $\begin{array}{l}8 \\
(18 \%)\end{array}$ & $\begin{array}{l}5 \\
(11 \%)\end{array}$ \\
\hline
\end{tabular}

Age of onset and age of diagnosis: $84 \%$ reported onset of symptoms before 10 years of age by comparison $49 \%$ were diagnosed after 30 years. 


\section{Table 2}

A summary of presenting history and clinical features

\begin{tabular}{|l|l|l|}
\hline History & Number Affected & Percentage of total $(\mathbf{n}=\mathbf{4 5})$ \\
\hline Exercise induced cramps/ myalgia & 45 & $100 \%$ \\
\hline Recognition by patient of second wind & 35 & $78 \%$ \\
\hline Myoglobinuria & 28 & $62 \%$ \\
\hline Acute renal failure & 5 & $11 \%$ \\
\hline Raised plasma urate / gout & 5 & $11 \%$ \\
\hline Chronic Fatigue & 18 & $40 \%$ \\
\hline Anxiety / depression / bipolar disorder & 14 & $31 \%$ \\
\hline Examination & & \\
\hline $\begin{array}{l}\text { Muscle hypertrophy (deltoid, biceps, } \\
\text { quads, calves) }\end{array}$ & 11 & \\
\hline $\begin{array}{l}\text { Muscle wasting (shoulder girdle, } \\
\text { paraspinals) }\end{array}$ & 15 & $33 \%$ \\
\hline Muscle weakness, shoulder girdle, axial & 7 & $16 \%$ \\
\hline
\end{tabular}




\section{Table 3}

\section{DNA analysis}

Relative frequency of mutated PYGM alleles. $96 \%$ of patients were found to carry at least one mutated allele for p.R50X or p.G205S.

\begin{tabular}{|c|c|c|c|}
\hline $\begin{array}{l}\text { p.R50X/ } \\
\text { p.R50X }\end{array}$ & p.R50X/ other mutation & $\begin{array}{l}\text { p.R50X/ } \\
\text { p.G205S }\end{array}$ & Other \\
\hline $\mathrm{N}=27$ & 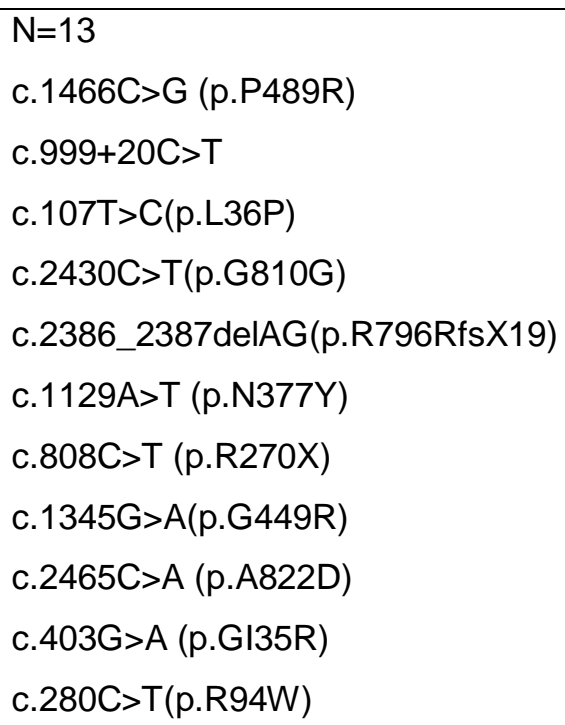 & $\mathrm{N}=3$ & $\begin{array}{l}\mathrm{N}=2 \\
\text { c.14delT/ c.14delT } \\
\text { (p.L5RfsX20/p.L5RfsX20 } \\
\text { c.107T>C/c.1239A+1G>A } \\
\text { (p.L36P/c.1239A+1G>A) }\end{array}$ \\
\hline $60 \%$ & $29 \%$ & $7 \%$ & $4 \%$ \\
\hline
\end{tabular}


Figure legends:

Figure 1a

Muscle biopsy of the patient with McArdle disease with atypical pathology showing rimmed vacuoles, abnormal variation in fibre size and several internal nuclei in addition to peripheral blebs of accumulated glycogen (a, Gomori trichrome stain); and several regenerating fibres that stain for the brain isoform of phosphorylase (c) and neonatal myosin (d). Immunolabelling of sarcolemmal proteins including dystrophin (b) was normal.

\section{Figure 1b}

Typical muscle biopsy from a different patient with McArdle Disease showing a) subsarcolemmal glycogen blebs and b) absent phosphorylase

\section{Figure 2}

\section{Figure 2 A}

Results of functional walking assessment $n=20$

Mean walking speed $\left(\mathrm{m} \cdot \mathrm{min}^{-1}\right)$ during a 12-minute walk test

Error bars $=$ Standard Error of the Mean 


\section{Figure 2B}

Mean rating of pain (RPP) to walking speed $\left(\mathrm{m}^{\mathrm{min}}{ }^{-1}\right)$ ratio.

Error bars $=$ standard error of the mean.

Figure 2c

Mean Heart rate during 12 minute walk test expressed as a percentage of peak HR corrected for age

\section{Graph 1}

Individual patient results for maximal distance walked during a 12 minute walk test 
Figure 1a
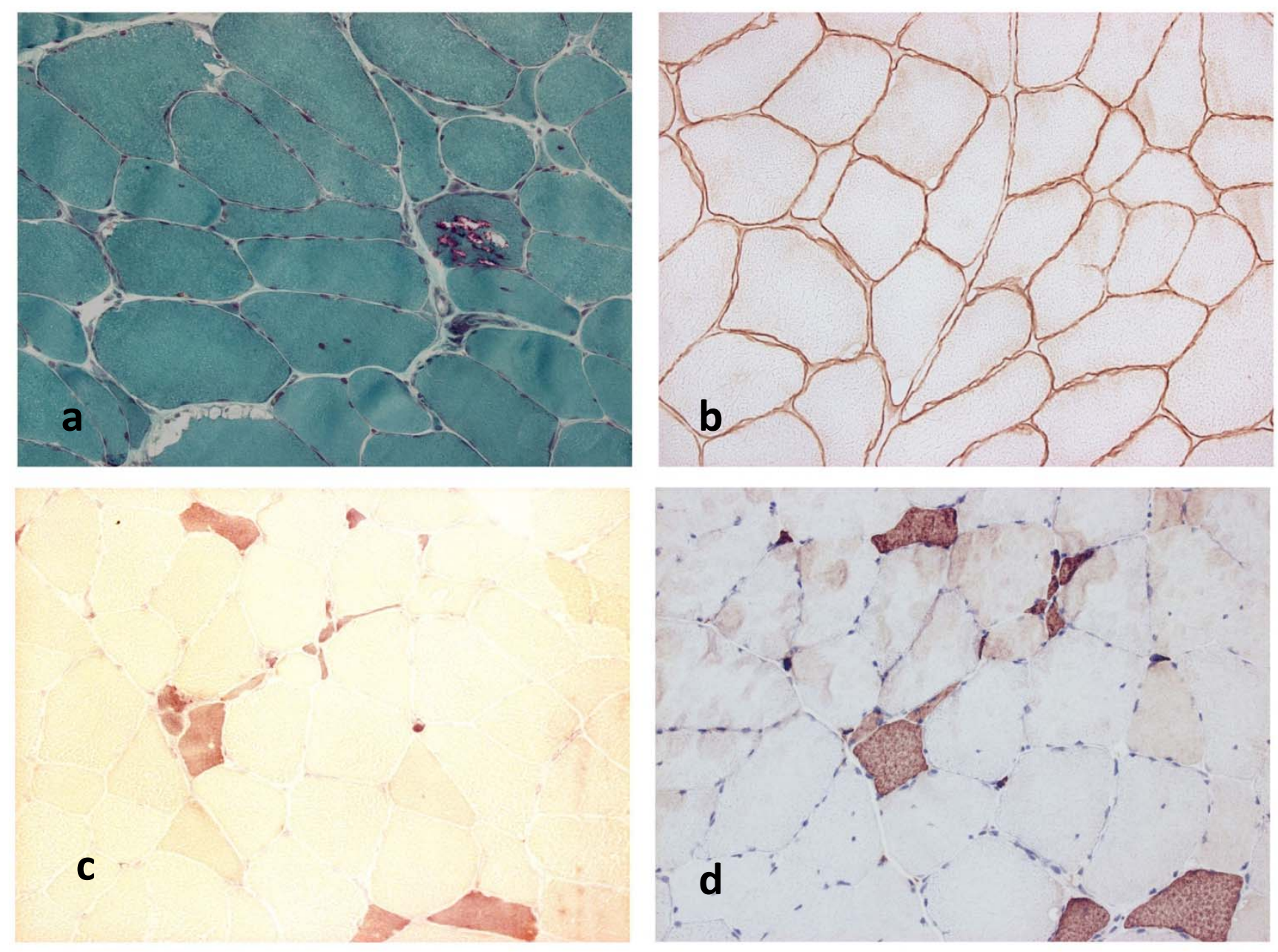
Figure 1b
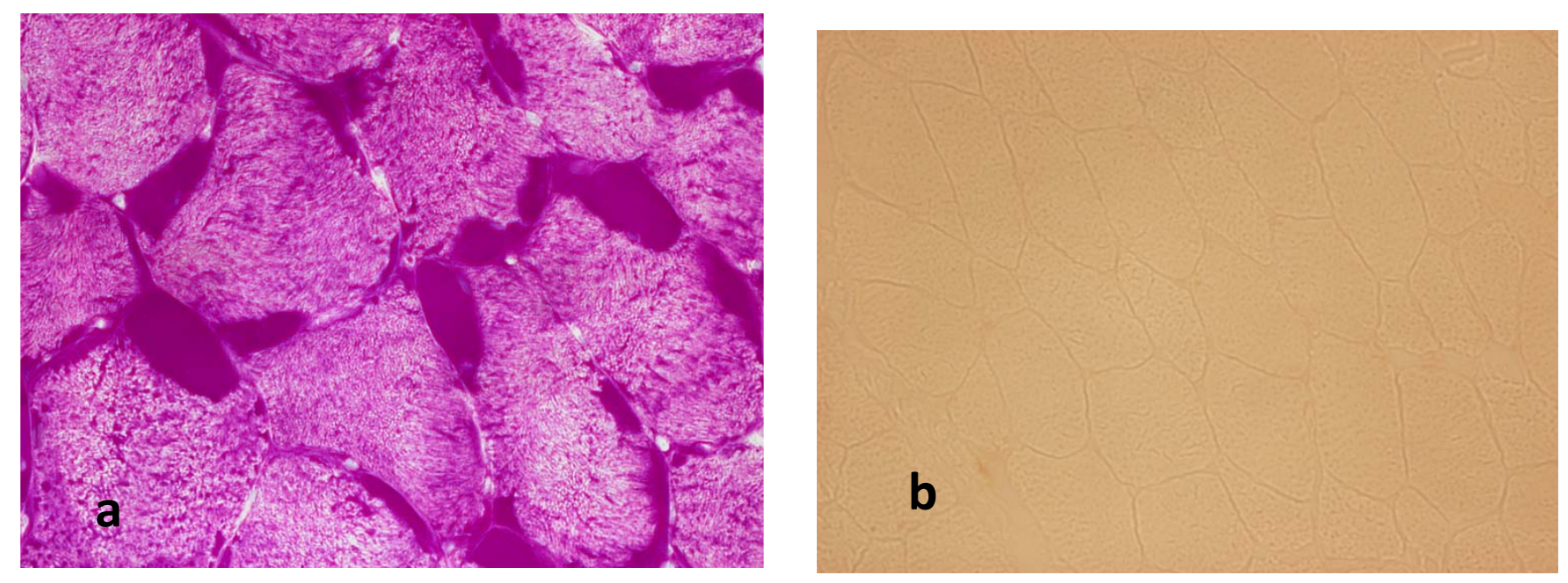
Figure 2a

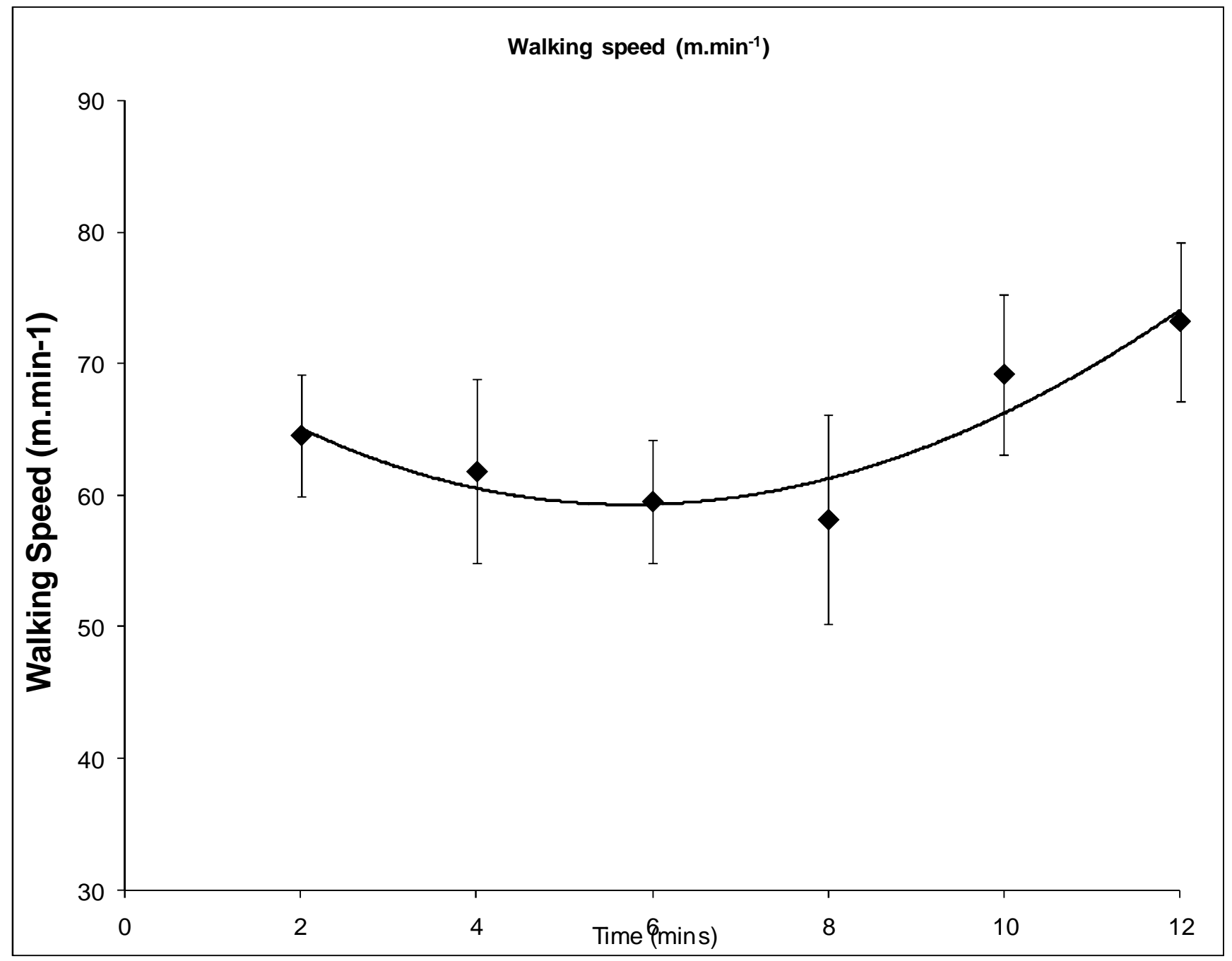


Figure $2 b$

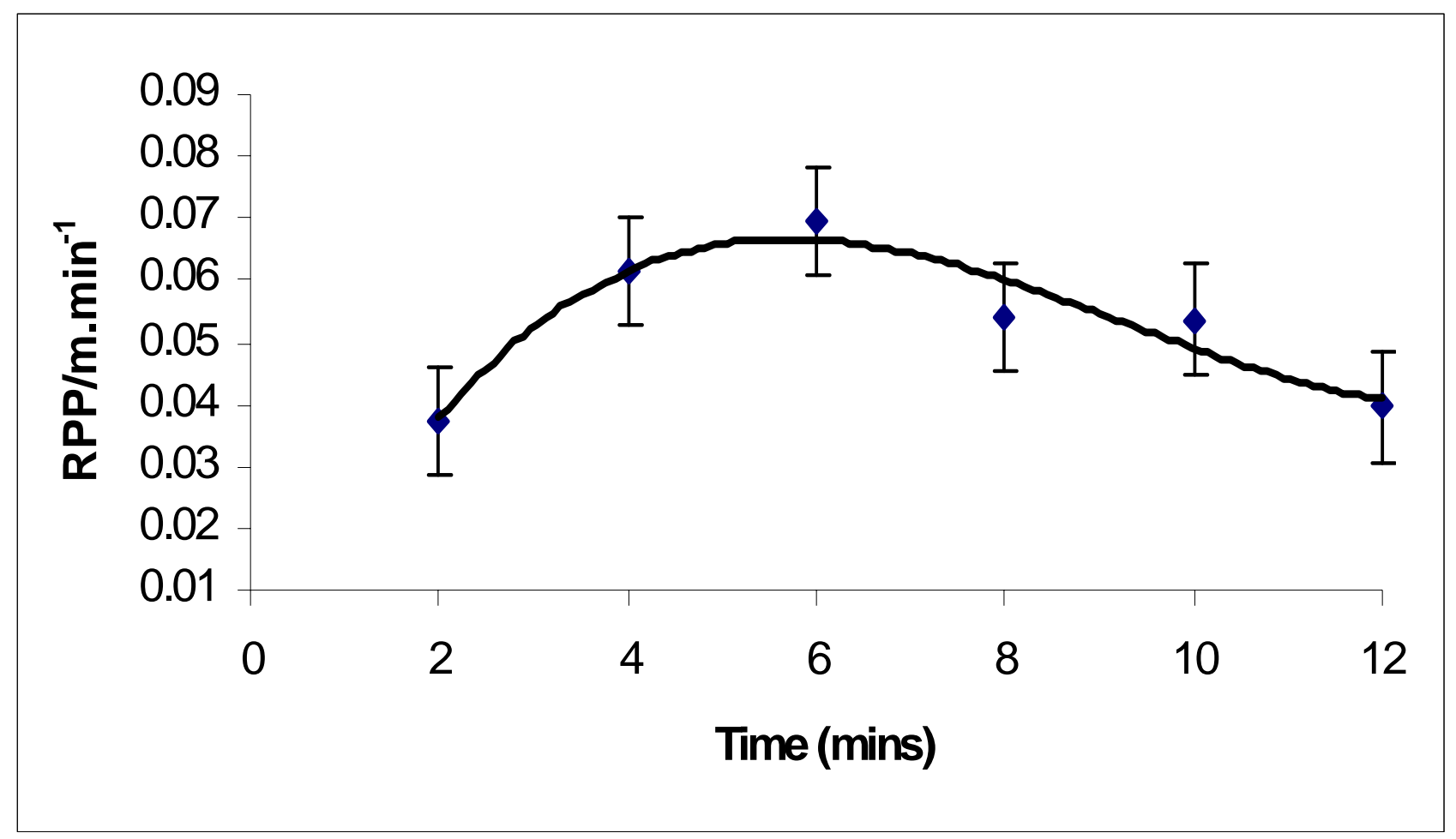


Figure 2c

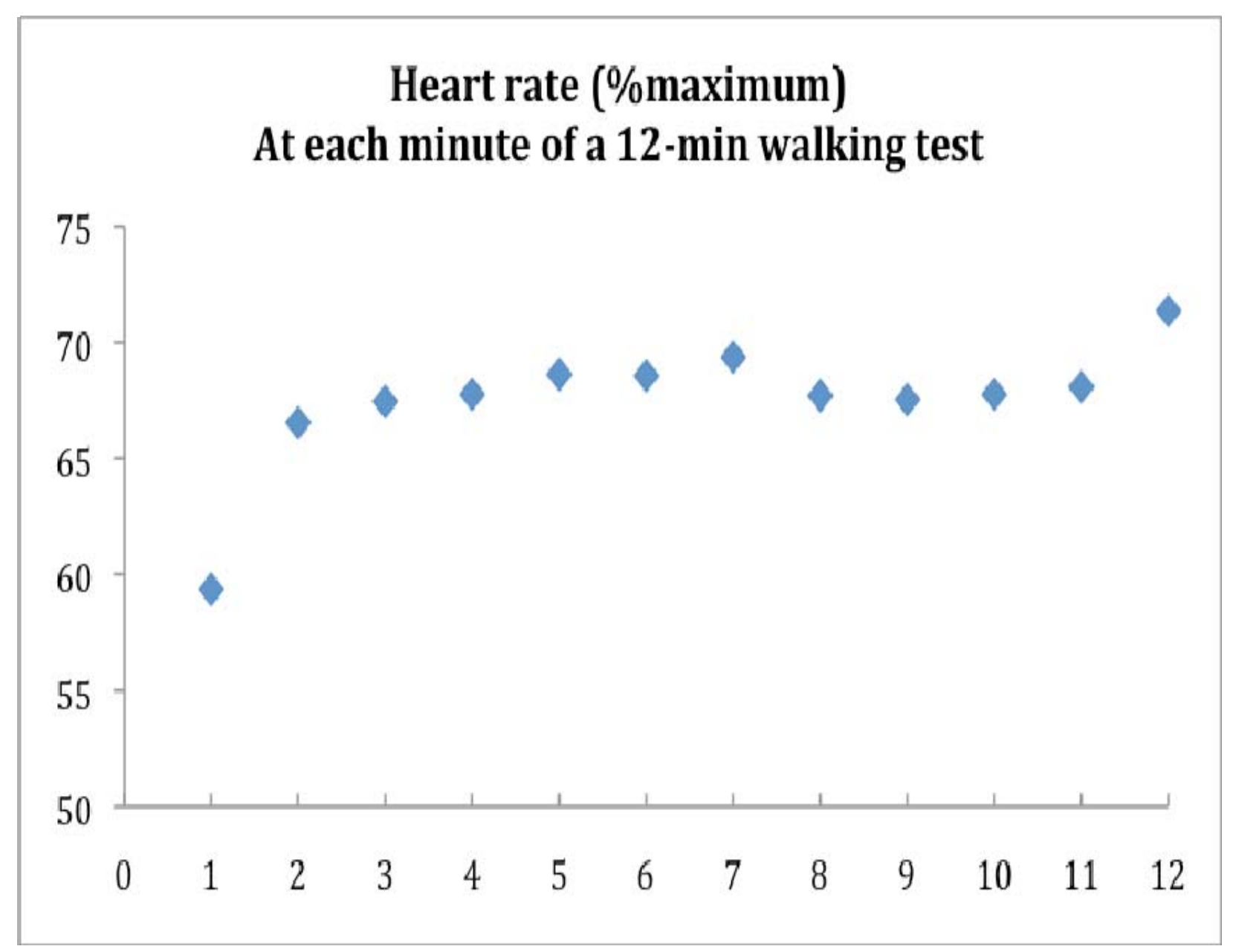


Graph 1

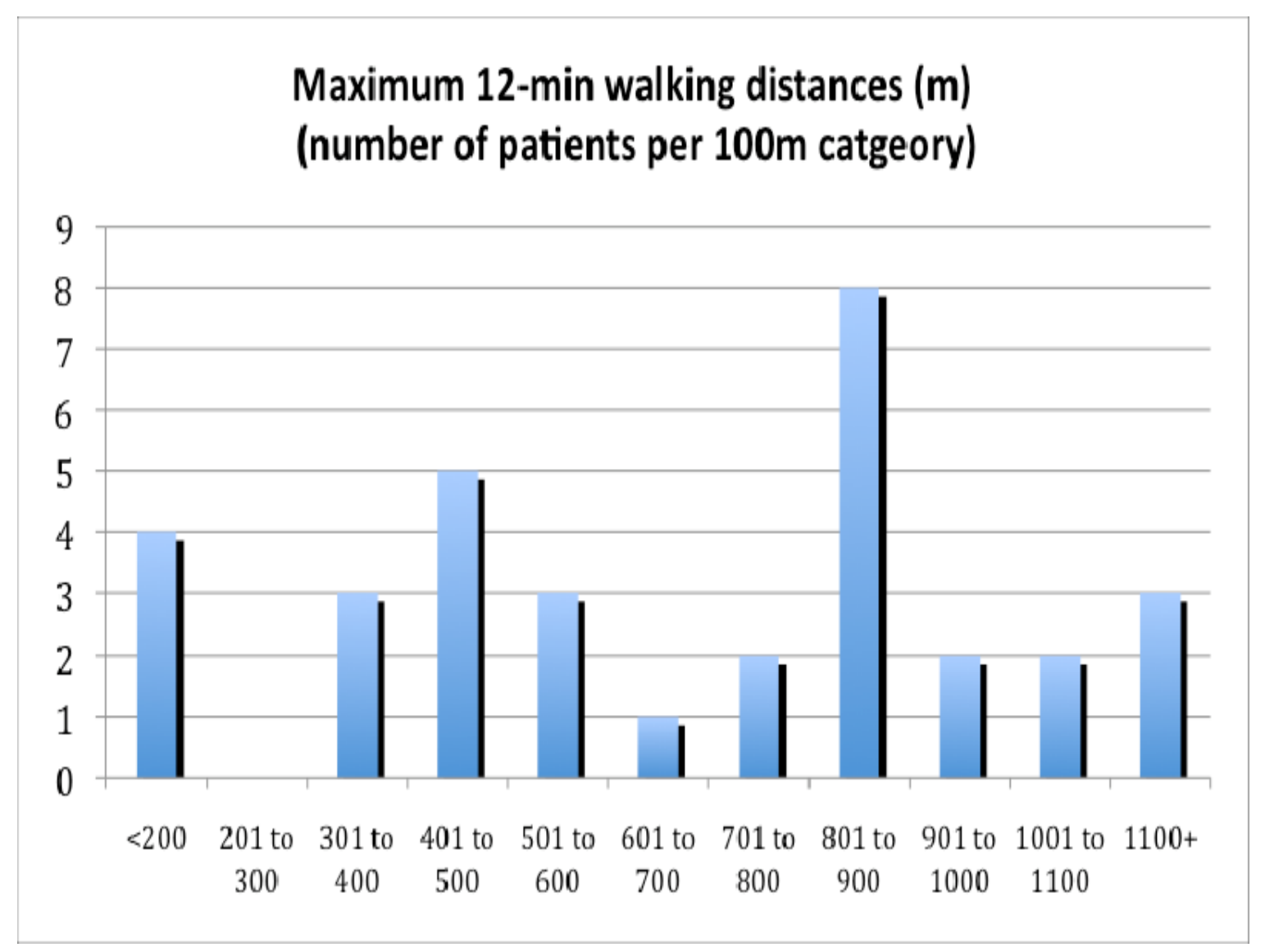

